\title{
Supplementary Information: GGL-Tox: Geometric Graph Learning for Toxicity Prediction
}

\author{
Jian Jiang ${ }^{1,2}$, Rui Wang ${ }^{2}$ and Guo-Wei Wei ${ }^{2,3,4 *}$ \\ ${ }^{1}$ Research Center of Nonlinear Science, College of Mathematics \\ and Computer Science, Engineering Research Center of Hubei \\ Province for Clothing Information, Wuhan Textile University, \\ Wuhan, 430200, P R. China \\ ${ }^{2}$ Department of Mathematics, Michigan State University, \\ East Lansing, 48824, Michigan \\ ${ }^{3}$ Department of Electrical and Computer Engineering, \\ Michigan State University, East Lansing, 48824, Michigan \\ ${ }^{4}$ Department of Biochemistry and Molecular Biology, Michigan \\ State University, East Lansing, 48824, Michigan
}

\section{S1 List of Tables}

Table S1. Number of data points per assay in Tox21 Challenge Data sets and the target and assay information.

Table S2. Sets of parameters in the three-scale GGL-Tox model for datasets in Tox21 Data Challenge 2014.

Table S3. List of element types in Tox21 Data Challenge 2014.

Table S4. A brief summary of three 2D molecular fingerprints.

Table S5. The hyper-parameters in GBDT algorithm with five different fingerprints for 12 datasets in toxicity prediction.

Table S6. The performance of test data and cross validation data for 12 datasets with the GGL-Tox model.

Table S7. Prediction results of GBDT with the Morgan fingerprint in Tox21 prediction.

Table S8. The hyper-parameters in GBDT algorithm with the Morgan fingerprint for 12 datasets in toxicity prediction.

Table S9. Comparison of prediction results of GBDT, RF, and SVM with the MWCG fingerprint in toxicity prediction.

Table S10. The main hyperparameters of RF in the implementation in toxicity prediction.

Table S11. The main hyperparameters of SVM in the implementation in toxicity prediction.

Table S12. Sample sizes for different datasets before and after data preprocessing.

Table S13. The omitted sample IDs in the training and test sets of 12 datasets. The numbers of omitted samples are given in the bracket.

Table S14. Comparison of prediction results of GBDT with five different molecular fingerprints in toxicity prediction before data preprocessing.

Table S15. The hyper-parameters in GBDT algorithm with five different fingerprints for 12 datasets in toxicity prediction before data preprocessing.

\footnotetext{
${ }^{*}$ Corresponding author. Email: wei@math.msu.edu
} 


\section{S2 Tox21 Data Challenge}

The Tox21 Data Challenge releases a data set with 12707 chemical compounds, including a training data set of 11764, a leaderboard set of 296, and a test set of 647 compounds. The training data set, referred as to Tox21_10k, is designed for model development and hyper-parameters turning against 12 assays. The leaderboard set, referred as to Tox21_LDB, is used to rank teams' submissions during the early phase of the competition. The test set, or the external validation set, referred as to Tox21_Ext_Valid, is employed for model evaluation and ranking the submissions at the final phase of the competition. The chemical compounds are given in the SDF format, which contains the chemical structure as undirected, labeled graphs whose nodes and edges represent atoms and bonds, respectively. The compounds are labeled according to the outcomes of the measurements as either "active" or "inactive" (while not all compounds are assessed by all assays). We download the Tox21 Challenge Data sets from wet site https://tripod.nih.gov/tox21/challenge/, including 7 NR pathway assays and 5 SR pathway assays. The number of active and inactive compounds in each data set, the number of compounds in train and test set, and the descriptions of the assay targets are shown in Table S1. It should be noted that the data sets in Table S1 were pre-processed prior to modeling steps.

Table S1. Number of data points per assay in Tox21 Challenge Data sets and the target and assay information.

\begin{tabular}{|l|l|c|c|c|c|}
\hline Dataset & Target/Assay & Train & Toxic/Non-toxic & Test & Toxic/non-toxic \\
\hline NR-AhR & aryl hydrocarbon receptor & 8162 & $950 / 7212$ & 609 & $73 / 536$ \\
\hline NR-AR & androgen receptor & 9353 & $380 / 8973$ & 585 & $12 / 573$ \\
\hline NR-AR-LBD & androgen receptor (luciferase assay) & 8591 & $303 / 8288$ & 581 & $8 / 573$ \\
\hline NR-Aromatase & aromatase & 7220 & $360 / 6860$ & 527 & $39 / 488$ \\
\hline NR-ER & estrogen receptor & 7689 & $937 / 6752$ & 515 & $51 / 464$ \\
\hline NR-ER-LBD & estrogen receptor (luciferase assay) & 8743 & $446 / 8297$ & 599 & $20 / 579$ \\
\hline NR-PPAR-gamma & $\begin{array}{l}\text { peroxisome proliferator-activated re- } \\
\text { ceptor gamma }\end{array}$ & 8176 & $221 / 7955$ & 604 & $31 / 573$ \\
\hline SR-ARE & $\begin{array}{l}\text { nuclear factor (erythroid- derived 2)- } \\
\text { like 2 antioxidant responsive element }\end{array}$ & 7163 & $1097 / 6066$ & 554 & $93 / 461$ \\
\hline SR-ATAD5 & genotoxicity indicated by ATAD5 & 9085 & $338 / 8747$ & 621 & $38 / 583$ \\
\hline SR-HSE & heat shock factor response element & 8144 & $427 / 7717$ & 609 & $22 / 587$ \\
\hline SR-MMP & mitochondrial membrane potentia & 7314 & $1142 / 6172$ & 542 & $60 / 482$ \\
\hline SR-p53 & DNA damage p53-pathway & 8626 & $537 / 8089$ & 615 & $41 / 574$ \\
\hline
\end{tabular}

Table S2. Sets of parameters in the three-scale GGL-Tox model for datasets in Tox21 Data Challenge 2014.

\begin{tabular}{|c|c|c|c|c|c|c|}
\hline Dataset & $\kappa_{1}$ & $\eta_{1}$ & $\nu_{2}$ & $\eta_{2}$ & $\nu_{3}$ & $\eta_{3}$ \\
\hline NR-AhR & 5.5 & 1.5 & 10 & 2 & 5.5 & 1 \\
\hline NR-AR & 15 & 1.5 & 6 & 1 & 5.5 & 0.5 \\
\hline NR-AR-LBD & 4.5 & 2 & 10 & 1.5 & 15 & 1 \\
\hline NR-Aromatase & 10 & 6 & 4 & 0.5 & 15 & 1 \\
\hline NR-ER & 1.5 & 3 & 6 & 1 & 5 & 3.5 \\
\hline NR-ER-LBD & 1 & 0.5 & 6 & 1 & 15 & 0.5 \\
\hline NR-ppar-gamma & 10 & 4.5 & 5.5 & 1 & 15 & 1 \\
\hline SR-ARE & 1 & 4 & 5.5 & 0.5 & 15 & 1 \\
\hline SR-ATAD5 & 3 & 1 & 10 & 1 & 15 & 1.5 \\
\hline SR-HSE & 3.5 & 3 & 20 & 1 & 15 & 1 \\
\hline SR-MMP & 10 & 3.5 & 5 & 1 & 20 & 1.5 \\
\hline SR-p53 & 1 & 1.5 & 10 & 1 & 15 & 1 \\
\hline
\end{tabular}


Table S3. List of element types in Tox21 Data Challenge 2014.

\begin{tabular}{|l|l|l|l|l|l|l|l|l|l|l|l|l|l|l|l|}
\hline $\mathrm{Cl}$ & $\mathrm{C}$ & $\mathrm{N}$ & $\mathrm{O}$ & $\mathrm{Br}$ & $\mathrm{Na}$ & $\mathrm{H}$ & $\mathrm{S}$ & $\mathrm{Hg}$ & $\mathrm{F}$ & $\mathrm{I}$ & $\mathrm{P}$ & $\mathrm{Ca}$ & $\mathrm{Pt}$ & $\mathrm{As}$ & $\mathrm{B}$ \\
\hline $\mathrm{Bi}$ & $\mathrm{Co}$ & $\mathrm{K}$ & $\mathrm{Fe}$ & $\mathrm{Gd}$ & $\mathrm{Zn}$ & $\mathrm{Al}$ & $\mathrm{Au}$ & $\mathrm{Si}$ & $\mathrm{Cu}$ & $\mathrm{Cr}$ & $\mathrm{Cd}$ & $\mathrm{V}$ & $\mathrm{Li}$ & $\mathrm{Se}$ & $\mathrm{Ag}$ \\
\hline $\mathrm{Ba}$ & $\mathrm{Ti}$ & $\mathrm{Tl}$ & $\mathrm{Sr}$ & $\mathrm{In}$ & $\mathrm{Dy}$ & $\mathrm{Ni}$ & $\mathrm{Be}$ & $\mathrm{Mg}$ & $\mathrm{Nd}$ & $\mathrm{Pd}$ & $\mathrm{Mn}$ & $\mathrm{Zr}$ & $\mathrm{Pb}$ & $\mathrm{Yb}$ & $\mathrm{Mo}$ \\
\hline $\mathrm{Sn}$ & $\mathrm{Eu}$ & $\mathrm{Sb}$ & $\mathrm{Sc}$ & $\mathrm{Ge}$ & & & & & & & & & & & \\
\hline
\end{tabular}

Table S4. A brief summary of three 2D molecular fingerprints.

\begin{tabular}{|c|l|c|}
\hline Fingerprint & Description & Number of features \\
\hline MACCS & $\begin{array}{l}\text { A substructure keys-based fingerprint with 166 structural keys } \\
\text { based on SMARTS patterns }\end{array}$ & 166 \\
\hline Estate 1 & $\begin{array}{l}\text { A topological fingerprint based on electro-topological state in- } \\
\text { dices, encoding the intrinsic electronic state of the atom as per- } \\
\text { turbed by the electronic influence of all other atoms in the } \\
\text { molecule within the context of the topological character of the } \\
\text { molecule. It means the number of times that each atom type is } \\
\text { hit }\end{array}$ & 79 \\
\hline Estate 2 & $\begin{array}{l}\text { Similar to Estate 1, and it contains the sum of the estate indices } \\
\text { for atoms of each type }\end{array}$ & 79 \\
\hline Morgan & $\begin{array}{l}\text { extended-connectivity fingerprint (ECFP), it is a well-known ex- } \\
\text { ample of circular fingerprints which are hashed topological fin- } \\
\text { gerprints but rather than looking for paths in a molecule and } \\
\text { record the environment of each atom up to a pre-determined ra- } \\
\text { dius. Morgan fingerprint with radius 2 is equivalent to ECFP4. }\end{array}$ & 1024 \\
\hline
\end{tabular}

\section{S3 Data preprocessing}

In the literature, different methods of data preprocessing are utilized in the studies of toxicity prediction of Tox21 Data Challenge 2014 by various authors. The sizes of processed datasets used in prediction algorithms are different, which greatly hinders the comparison of predictive performance of different algorithms in these studies. For instance, the range of sample numbers in Ref. [1] is from 5747 for NR-Aromatase to 6950 for NR-AR, while in Ref. [2], this range is from 7105 to 9219 for the same dataset. In Ref. [3], this range changes from 7455 for SR-ARE to 9609 for NR-AR. Andreas et al. used deep learning for toxicity prediction in Tox21 Data Challenge, where 12707 compounds were reduced to 8694 distinct fragments [4]. In Reference [5], three assays, NR-AhR, NR-ER-LBD, and SR-HSE, were used for toxicity prediction, where the total compounds number for each assay is 6901,6801 , and 7328 respectively. These numbers are much smaller than 8457, 9016, and 8473 in our work. Additionally, in Reference [6], after data curation, the size of the training set, test set 1 , and test set 2 were reduced from the original compound number 11764, 296, and 647, to 9323, 291, and 641, respectively. Another problem is that the IDs of omitted samples were not given in the literature. Therefore, in present work, in order to compare the performance of our model with other start-of-the-art ML algorithms reported in Ref. [3], we have chosen the same sample size. The original sample sizes of train and test sets for each dataset can be found in Table S1 and the adjusted train and test sets for each dataset are shown in Table S12. To ensure the reproducibility and promote fair comparison, the omitted compound IDs in train and test sets of 12 datasets are given in Table S13.

About the standardization process of data, it contains several options, such as removing salts and solvents, disconnecting metal atoms, removing fragments, adding explicit hydrogen atoms, aiming to retain only suitable structures of compounds that may have an unknown influence on the prediction accuracy. In the present work, to keep the data integrity and the comparison fairness with other works to the maximum extent, we do not do the standardization process on compounds before the fingerprints generation.

We implement the predictive performance of 12 datasets before the data preprocessing in Table S14 and 
Table S5. The hyper-parameters in GBDT algorithm with five different fingerprints for 12 datasets in toxicity prediction.

\begin{tabular}{|c|c|c|c|c|c|c|c|c|c|c|c|}
\hline Dataset & $\begin{array}{l}\text { finger } \\
\text { print }\end{array}$ & $\begin{array}{l}n \text { es- } \\
\text { tima- } \\
\text { tors }\end{array}$ & $\begin{array}{l}\max \\
\text { depth }\end{array}$ & $\begin{array}{l}\text { min } \\
\text { sam- } \\
\text { ple } \\
\text { split }\end{array}$ & $\begin{array}{l}\text { learning } \\
\text { rate }\end{array}$ & Dataset & $\begin{array}{l}\text { finger } \\
\text { print }\end{array}$ & $\begin{array}{l}\mathrm{n} \text { es- } \\
\text { tima- } \\
\text { tors }\end{array}$ & $\begin{array}{l}\max \\
\text { depth }\end{array}$ & $\begin{array}{l}\text { min } \\
\text { sam- } \\
\text { ple } \\
\text { split }\end{array}$ & $\begin{array}{l}\text { learning } \\
\text { rate }\end{array}$ \\
\hline \multirow{5}{*}{$\begin{array}{l}\text { NR- } \\
\text { AhR }\end{array}$} & Estate 2 & 13000 & 9 & 8 & 0.005 & \multirow{5}{*}{$\begin{array}{l}\text { NR- } \\
\text { ppar- } \\
\text { gamma }\end{array}$} & Estate 2 & 9000 & 3 & 6 & 0.02 \\
\hline & Estate 1 & 8000 & 7 & 2 & 0.005 & & Estate 1 & 12000 & 3 & 2 & 0.02 \\
\hline & MACCS & 8000 & 9 & 4 & 0.001 & & MACCS & 9000 & 3 & 8 & 0.0005 \\
\hline & MWCG & 10000 & 3 & 3 & 0.005 & & MWCG & 10000 & 3 & 3 & 0.005 \\
\hline & Hybrid & 10000 & 5 & 2 & 0.008 & & Hybrid & 8000 & 7 & 2 & 0.01 \\
\hline \multirow{5}{*}{ NR-AR } & Estate 2 & 15000 & 5 & 8 & 0.0001 & \multirow{5}{*}{$\begin{array}{l}\text { SR- } \\
\text { ARE }\end{array}$} & Estate 2 & 8000 & 9 & 8 & 0.0005 \\
\hline & Estate 1 & 15000 & 7 & 2 & 0.003 & & Estate 1 & 9000 & 7 & 8 & 0.0005 \\
\hline & MACCS & 10000 & 3 & 2 & 0.0001 & & MACCS & 8000 & 9 & 2 & 0.0005 \\
\hline & MWCG & 15000 & 7 & 6 & 0.005 & & MWCG & 8000 & 3 & 4 & 0.02 \\
\hline & Hybrid & 8000 & 5 & 6 & 0.0001 & & Hybrid & 12000 & 9 & 8 & 0.02 \\
\hline \multirow{5}{*}{$\begin{array}{l}\text { NR- } \\
\text { AR- } \\
\text { LBD }\end{array}$} & Estate 2 & 11000 & 3 & 6 & 0.005 & \multirow{5}{*}{$\begin{array}{l}\text { SR- } \\
\text { ATAD5 }\end{array}$} & Estate 2 & 15000 & 9 & 4 & 0.0005 \\
\hline & Estate 1 & 9000 & 9 & 4 & 0.0001 & & Estate 1 & 15000 & 5 & 6 & 0.007 \\
\hline & MACCS & 8000 & 9 & 4 & 0.003 & & MACCS & 10000 & 7 & 4 & 0.0005 \\
\hline & MWCG & 8000 & 5 & 3 & 0.005 & & MWCG & 12000 & 7 & 6 & 0.008 \\
\hline & Hybrid & 8000 & 9 & 8 & 0.008 & & Hybrid & 8000 & 5 & 6 & 0.008 \\
\hline \multirow{5}{*}{$\begin{array}{l}\text { NR- } \\
\text { Arom } \\
\text { atase }\end{array}$} & Estate 2 & 12000 & 5 & 6 & 0.0001 & \multirow{5}{*}{$\begin{array}{l}\text { SR- } \\
\text { HSE }\end{array}$} & Estate 2 & 15000 & 9 & 2 & 0.02 \\
\hline & Estate 1 & 11000 & 5 & 8 & 0.0005 & & Estate 1 & 15000 & 3 & 6 & 0.001 \\
\hline & MACCS & 9000 & 9 & 2 & 0.001 & & MACCS & 11000 & 7 & 8 & 0.0001 \\
\hline & MWCG & 15000 & 7 & 2 & 0.008 & & MWCG & 12000 & 7 & 9 & 0.005 \\
\hline & Hybrid & 15000 & 5 & 4 & 0.0001 & & Hybrid & 12000 & 3 & 8 & 0.01 \\
\hline \multirow{5}{*}{ NR-ER } & Estate 2 & 12000 & 3 & 4 & 0.0005 & \multirow{5}{*}{$\begin{array}{l}\text { SR- } \\
\text { MMP }\end{array}$} & Estate 2 & 8000 & 9 & 2 & 0.001 \\
\hline & Estate 1 & 15000 & 5 & 2 & 0.001 & & Estate 1 & 8000 & 5 & 2 & 0.007 \\
\hline & MACCS & 15000 & 7 & 6 & 0.01 & & MACCS & 9000 & 9 & 6 & 0.003 \\
\hline & MWCG & 10000 & 3 & 3 & 0.005 & & MWCG & 10000 & 7 & 3 & 0.01 \\
\hline & Hybrid & 8000 & 7 & 8 & 0.008 & & Hybrid & 8000 & 5 & 4 & 0.01 \\
\hline \multirow{5}{*}{$\begin{array}{l}\text { NR- } \\
\text { ER- } \\
\text { LBD }\end{array}$} & Estate 2 & 9000 & 9 & 2 & 0.0005 & \multirow{5}{*}{ SR-p53 } & Estate 2 & 8000 & 9 & 2 & 0.0005 \\
\hline & Estate 1 & 8000 & 5 & 6 & 0.003 & & Estate 1 & 9000 & 7 & 4 & 0.001 \\
\hline & MACCS & 10000 & 3 & 4 & 0.0005 & & MACCS & 8000 & 7 & 2 & 0.001 \\
\hline & MWCG & 10000 & 7 & 6 & 0.005 & & MWCG & 8000 & 5 & 3 & 0.005 \\
\hline & Hybrid & 10000 & 9 & 4 & 0.01 & & Hybrid & 10000 & 9 & 2 & 0.008 \\
\hline
\end{tabular}

Table S6. The performance of test data and cross validation (CV) data for 12 datasets with GGL-Tox method. The numbers from 1 to 12 in the column of Dataset correspond to Tox21 datasets of NR-AhR, NR-AR, NR-AR-LBD, NR-Aromatase, NR-ER, NR-ER-LBD, NR-PPAR-gamma, SR-ARE, SR-ATAD5, SR-HSE, SR-MMP, and SR-p53, respectively.

\begin{tabular}{|c|c|c|c|c|c|c|c|c|c|c|c|c|}
\hline & 1 & 2 & 3 & 4 & 5 & 6 & 7 & 8 & 9 & 10 & 11 & 12 \\
\hline AUC (test data) & 0.887 & 0.915 & 0.991 & 0.834 & 0.836 & 0.892 & 0.773 & 0.803 & 0.833 & 0.978 & 0.931 & 0.810 \\
\hline AUC (CV data) & 0.893 & 0.849 & 0.899 & 0.962 & 0.801 & 0.974 & 0.893 & 0.842 & 0.855 & 0.907 & 0.959 & 0.870 \\
\hline
\end{tabular}

Table S7. Prediction results of GBDT with the Morgan fingerprint in Tox21 prediction. The performance of the model is evaluated by the accuracy (ACC), ROC-curve (AUC), balanced accuracy (BA), and Matthews correlation coefficient (MCC). The numbers from 1 to 12 in the column of Dataset correspond to Tox21 datasets of NR-AhR, NR-AR, NR-AR-LBD, NR-Aromatase, NR-ER, NR-ER-LBD, NR-PPAR-gamma, SR-ARE, SR-ATAD5, SR-HSE, SR-MMP, and SR-p53, respectively.

\begin{tabular}{|c|c|c|c|c|c|c|c|c|c|c|c|c|}
\hline metrics & 1 & 2 & 3 & 4 & 5 & 6 & 7 & 8 & 9 & 10 & 11 & 12 \\
\hline ACC & 0.877 & 0.979 & 0.984 & 0.930 & 0.907 & 0.971 & 0.948 & 0.815 & 0.939 & 0.958 & 0.893 & 0.932 \\
\hline AUC & 0.832 & 0.683 & 0.781 & 0.643 & 0.746 & 0.802 & 0.736 & 0.689 & 0.714 & 0.722 & 0.872 & 0.752 \\
\hline BA & 0.592 & 0.551 & 0.499 & 0.536 & 0.585 & 0.563 & 0.525 & 0.536 & 0.523 & 0.529 & 0.660 & 0.520 \\
\hline MCC & 0.276 & 0.208 & -0.004 & 0.231 & 0.291 & 0.330 & 0.127 & 0.104 & 0.116 & 0.090 & 0.377 & 0.070 \\
\hline
\end{tabular}

the values of hyper-parameters for each dataset are shown in Table S15. The highlight part in Table S14 is the optimal AUC values among five fingerprints. From this table, we can find that for Estate 2 fingerprint, it corresponds to best performance in one assay, like NR-ER-LBD; Estate 1 fingerprint also corresponds to best performance in one assay, like NR-AR; MACCS fingerprint makes three assays best, like NR-AR-LBD, NR-ppar-gamma and SR-HSE; MWCG fingerprint corresponds to two assays with best performance, like 
Table S8. The hyper-parameters in GBDT algorithm with the Morgan fingerprint for 12 datasets in toxicity prediction. The numbers from 1 to 12 in the column of Dataset correspond to Tox21 datasets of NR-AhR, NR-AR, NR-AR-LBD, NR-Aromatase, NR-ER, NRER-LBD, NR-PPAR-gamma, SR-ARE, SR-ATAD5, SR-HSE, SR-MMP, and SR-p53, respectively.

\begin{tabular}{|c|c|c|c|c|c|c|c|c|c|c|c|c|}
\hline hyper-parameters & 1 & 2 & 3 & 4 & 5 & 6 & 7 & 8 & 9 & 10 & 11 & 12 \\
\hline n estimators & 12000 & 9000 & 9000 & 15000 & 12000 & 9000 & 11000 & 15000 & 15000 & 9000 & 11000 & 15000 \\
\hline max depth & 5 & 7 & 3 & 9 & 5 & 3 & 5 & 7 & 9 & 7 & 3 & 9 \\
\hline min sample split & 8 & 8 & 8 & 8 & 4 & 8 & 8 & 8 & 4 & 8 & 8 & 4 \\
\hline learning rate & 0.005 & 0.001 & 0.001 & 0.003 & 0.001 & 0.001 & 0.0005 & 0.001 & 0.005 & 0.003 & 0.003 & 0.001 \\
\hline
\end{tabular}

Table S9. Comparison of prediction results of GBDT, RF, and SVM with the MWCG fingerprint in toxicity prediction.

\begin{tabular}{|c|c|c|c|c|c|c|}
\hline \multirow{2}{*}{ Dataset } & \multicolumn{5}{|c|}{ Algorithm } \\
\cline { 2 - 7 } & \multicolumn{2}{|c|}{ GBDT } & \multicolumn{2}{c|}{ RF } & \multicolumn{2}{c|}{ SVM } \\
\cline { 2 - 7 } & AUC & ACC & AUC & ACC & AUC & ACC \\
\hline NR-AhR & $\mathbf{0 . 8 8 7}$ & 0.916 & 0.882 & 0.906 & 0.873 & 0.902 \\
\hline NR-AR & $\mathbf{0 . 9 1 5}$ & 0.993 & 0.815 & 0.997 & 0.863 & 0.997 \\
\hline NR-AR-LBD & $\mathbf{0 . 9 9 1}$ & 0.995 & 0.920 & 0.996 & 0.846 & 0.996 \\
\hline NR-Aromatase & $\mathbf{0 . 8 3 4}$ & 0.950 & 0.729 & 0.950 & 0.718 & 0.948 \\
\hline NR-ER & $\mathbf{0 . 8 3 6}$ & 0.927 & 0.805 & 0.923 & 0.754 & 0.919 \\
\hline NR-ER-LBD & $\mathbf{0 . 8 9 2}$ & 0.988 & 0.870 & 0.988 & 0.889 & 0.990 \\
\hline NR-PPAR-gamma & $\mathbf{0 . 7 7 3}$ & 0.968 & 0.763 & 0.961 & 0.731 & 0.941 \\
\hline SR-ARE & $\mathbf{0 . 8 0 3}$ & 0.856 & 0.773 & 0.843 & 0.748 & 0.854 \\
\hline SR-ATAD5 & $\mathbf{0 . 8 3 3}$ & 0.962 & 0.755 & 0.962 & 0.711 & 0.962 \\
\hline SR-HSE & $\mathbf{0 . 9 7 8}$ & 0.985 & 0.942 & 0.987 & 0.897 & 0.988 \\
\hline SR-MMP & $\mathbf{0 . 9 3 1}$ & 0.913 & 0.916 & 0.924 & 0.846 & 0.896 \\
\hline SR-p53 & 0.810 & 0.963 & $\mathbf{0 . 8 6 2}$ & 0.958 & 0.857 & 0.955 \\
\hline
\end{tabular}

Table S10. The main hyperparameters of RF in the implementation in toxicity prediction.

\begin{tabular}{|c|c|c|c|c|}
\hline \multirow{2}{*}{ Dataset } & \multicolumn{4}{|c|}{ Main hyperparameters } \\
\cline { 2 - 5 } & Criterion & max_depth & max_features & n_estimators \\
\hline NR-AhR & entropy & 10 & 439 & 3236 \\
\hline NR-AR & gini & 1 & 911 & 1423 \\
\hline NR-AR-LBD & gini & 37 & 928 & 3455 \\
\hline NR-Aromatase & gini & 16 & 843 & 2848 \\
\hline NR-ER & entropy & 15 & 11 & 123 \\
\hline NR-ER-LBD & gini & 34 & 503 & 1867 \\
\hline NR-PPAR-gamma & gini & 40 & 367 & 1013 \\
\hline SR-ARE & entropy & 12 & 531 & 3931 \\
\hline SR-ATAD5 & gini & 5 & 654 & 2810 \\
\hline SR-HSE & entropy & 7 & 979 & 3417 \\
\hline SR-MMP & gini & 10 & 581 & 2848 \\
\hline SR-p53 & entropy & 31 & 791 & 663 \\
\hline
\end{tabular}

NR-AhR and SR-MMP, and for the hybrid fingerprint, it corresponds to five assays with best performance, like NR-Aromatase, NR-ER, SR-ARE, SR-ATAD5, and SR-p53. Additionally, through the comparison with Table 1 in the main text, we can see that the AUC value with best performance for each dataset before data preprocessing is smaller than that after the data curation. In other words, the data preprocessing procedure is necessary and could improve the predictive performance of ML algorithm. 
Table S11. The main hyperparameters of SVM in the implementation in toxicity prediction.

\begin{tabular}{|c|c|c|c|}
\hline \multirow{2}{*}{ Dataset } & \multicolumn{3}{|c|}{ Main hyperparameters } \\
\cline { 2 - 4 } & $C$ & gamma & kernel \\
\hline NR-AhR & 97.4968 & 0.0556 & poly \\
\hline NR-AR & 4.5498 & 0.0033 & rbf \\
\hline NR-AR-LBD & 93.1060 & 0.0327 & rbf \\
\hline NR-Aromatase & 1.9330 & 0.0025 & rbf \\
\hline NR-ER & 22.4327 & 0.0852 & rbf \\
\hline NR-ER-LBD & 9.8755 & 0.0193 & rbf \\
\hline NR-PPAR-gamma & 12.9143 & 0.0046 & rbf \\
\hline SR-ARE & 84.5949 & 0.0490 & poly \\
\hline SR-ATAD5 & 19.6794 & 0.0628 & poly \\
\hline SR-HSE & 2.5326 & 0.0049 & poly \\
\hline SR-MMP & 99.1062 & 0.0987 & rbf \\
\hline SR-p53 & 28.8880 & 0.0114 & rbf \\
\hline
\end{tabular}

Table S12. Sample sizes for different datasets before and after data preprocessing.

\begin{tabular}{|l|l|l|l|l|l|l|}
\hline \multirow{2}{*}{ Dataset } & \multicolumn{3}{|c|}{ Before } & \multicolumn{3}{c|}{ After } \\
\cline { 2 - 7 } & total size & train & test & total size & train & test \\
\hline NR-AhR & 8771 & 8162 & 609 & 8457 & 7863 & 594 \\
\hline NR-AR & 9938 & 9353 & 585 & 9609 & 9036 & 573 \\
\hline NR-AR-LBD & 9172 & 8591 & 581 & 8801 & 8234 & 567 \\
\hline NR-Aromatase & 7747 & 7220 & 527 & 7474 & 6959 & 515 \\
\hline NR-ER & 8204 & 7689 & 515 & 7926 & 7421 & 505 \\
\hline NR-ER-LBD & 9342 & 8743 & 599 & 9016 & 8431 & 585 \\
\hline NR-ppar-gamma & 8780 & 8176 & 604 & 8473 & 7883 & 590 \\
\hline SR-ARE & 7717 & 7163 & 554 & 7455 & 6915 & 540 \\
\hline SR-ATAD5 & 9706 & 9085 & 621 & 9381 & 8775 & 606 \\
\hline SR-HSE & 8753 & 8144 & 609 & 8473 & 7879 & 594 \\
\hline SR-MMP & 7856 & 7314 & 542 & 7601 & 7071 & 530 \\
\hline SR-p53 & 9241 & 8626 & 615 & 8950 & 8349 & 601 \\
\hline
\end{tabular}


Table S13. The omitted sample IDs in the training and test sets of 12 datasets. The numbers of omitted samples are given in the bracket.

\begin{tabular}{|c|c|c|}
\hline Dataset & & Omitted sample ID \\
\hline \multirow[t]{2}{*}{ NR-AhR } & $\begin{array}{c}\text { Training } \\
\text { (299) }\end{array}$ & 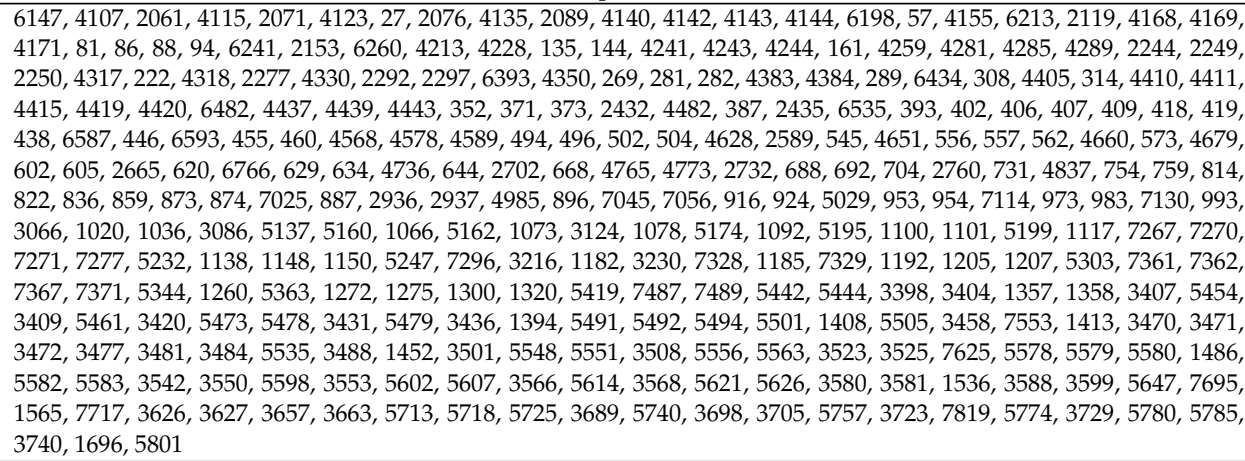 \\
\hline & Test(15) & $2,259,519,7,137,523,12,139,141,533,540,156,295,49,50$ \\
\hline \multirow[t]{2}{*}{ NR-AR } & $\begin{array}{c}\text { Training } \\
(317)\end{array}$ & 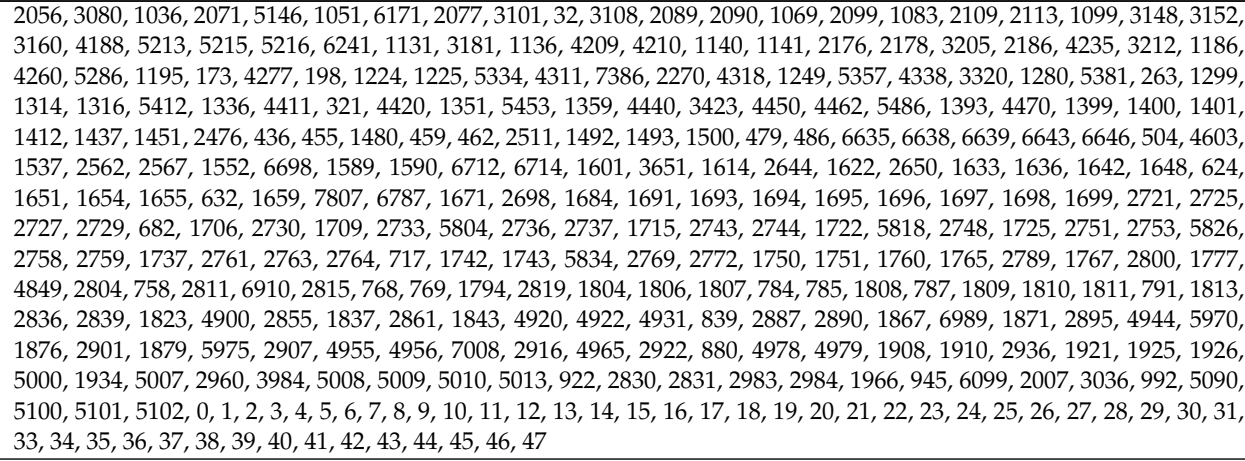 \\
\hline & Test(12) & $2,323,577,424,425,552,554,558,404,511,497,575$ \\
\hline \multirow[t]{2}{*}{ NR-AR-LBD } & $\begin{array}{c}\text { Training } \\
(357)\end{array}$ & 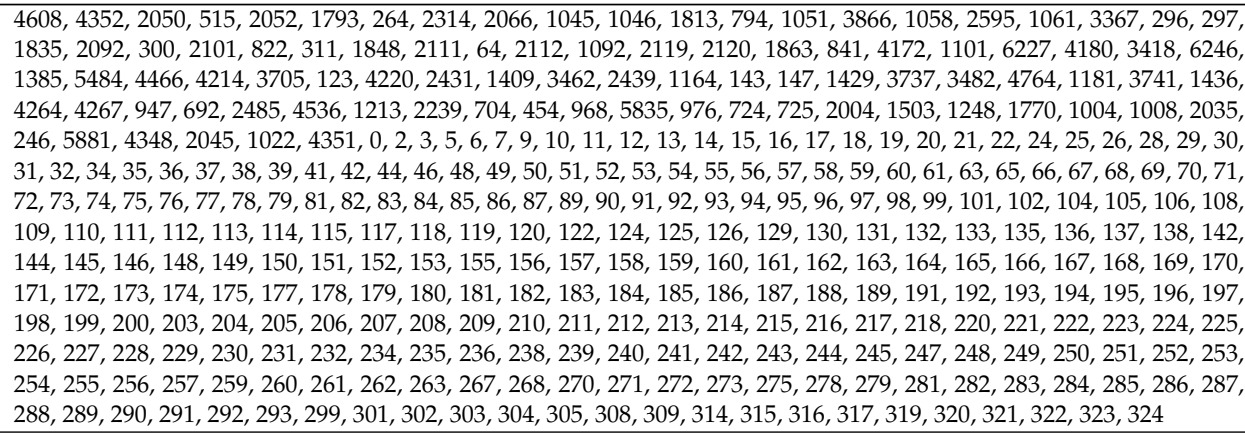 \\
\hline & Test(14) & $548,16,368,565,118,119,441,506,316,0,1,2,472,137$ \\
\hline \multirow[t]{2}{*}{ NR-Aromatase } & $\begin{array}{c}\text { Training } \\
\text { (261) }\end{array}$ & $\begin{array}{l}0,1,2562,4,5,6,8,3595,528,1552,18,533,534,25,27,31,32,544,2081,38,39,3626,43,5167,560,49,51,3124,53, \\
566,59,60,2109,6204,66,68,581,70,583,74,76,589,593,3155,84,5204,87,91,92,4699,94,606,4702,4703,1635, \\
1636,613,103,617,106,108,109,111,115,116,628,126,127,128,129,2688,3713,4735,645,134,135,2694,650,144, \\
2712,155,157,159,4772,165,679,168,682,173,686,4783,176,177,688,180,2740,182,183,2741,2745,2746,2747, \\
2751,2752,706,1731,2755,2756,2757,2758,3267,4808,5320,3275,204,1744,2769,3792,3283,725,214,3287,218, \\
219,2782,1762,3298,2788,2278,231,2790,4842,2796,3310,1263,3313,1267,1268,1269,1270,2804,760,249,2809, \\
251,252,3318,2814,3320,2816,4861,264,2824,266,2826,3341,1294,786,276,1302,3352,281,796,286,287,288,291, \\
293,294,1318,1319,3366,5420,2866,1333,2872,1337,4922,1343,320,2881,3904,5443,4932,325,837,1351,1352, \\
1353,4424,1358,847,2895,339,1364,854,343,4956,352,353,2914,357,358,873,362,364,365,372,1396,1397,376, \\
377,889,2430,2945,387,1415,393,394,396,4492,400,1424,3474,5017,3996,429,432,6576,1459,440,3521,964,\end{array}$ \\
\hline & Test(12) & $128,1,130,3,4,390,7,137,12,156,289,43$ \\
\hline
\end{tabular}




\begin{tabular}{|c|c|c|}
\hline Dataset & & Omitted sample ID \\
\hline \multirow[t]{2}{*}{ NR-ER } & $\begin{array}{c}\text { Training } \\
\text { (267) }\end{array}$ & $\begin{array}{l}0,2049,4097,3,2055,11,13,15,20,2069,22,2072,2073,26,28,2079,4130,2084,4132,39,6188,6189,50,51,2100, \\
4148,2105,6201,59,60,61,65,70,2121,4170,77,78,79,2125,81,2127,4176,84,87,4187,93,2141,4198,107,2157, \\
111,112,128,4226,131,4229,4234,6286,4244,6294,151,2208,2209,164,167,2223,183,185,186,187,188,6331,6336, \\
194,2245,4297,212,6359,223,224,226,6374,231,232,235,236,238,2286,240,242,243,245,2301,255,2303,257, \\
2307,260,271,274,281,282,4378,2335,291,2339,296,300,301,302,4403,4415,324,326,328,331,332,4430,336,338, \\
339,340,341,345,4442,2395,6492,6493,351,2399,354,2409,362,365,366,4462,371,372,373,4470,377,381,4477, \\
383,395,2443,4495,4496,4497,403,404,6548,6552,413,2476,430,6577,2483,442,443,6592,6597,2516,4574,488, \\
489,2545,6649,4602,2554,2555,2571,4623,2578,6682,4635,542,544,545,2594,6690,2596,2598,556,562,571,574, \\
2623,577,6724,581,2629,6727,584,2634,4684,6735,2640,599,6746,606,4703,608,615,616,623,624,625,4728, \\
6781,639,2692,2693,650,652,2702,4755,2723,677,681,2733,687,2737,691,2739,6839,695,696,697,4793,702, \\
4798,4801,706,707,6853,6854,711,2763,721,723,4820,4821,2776,732,733,736,737,740,747,748,749,750,751, \\
752,753,756,2807,4855,4857,6909,766,6913,774,777,780,781\end{array}$ \\
\hline & Test(10) & $128,2,131,5,6,7,136,261,10,262$ \\
\hline \multirow[t]{2}{*}{ NR-ER-LBD } & $\begin{array}{c}\text { Training } \\
\text { (312) }\end{array}$ & $\begin{array}{l}513,5122,1028,1031,8,520,2569,2059,12,3592,6159,1554,6162,2069,24,537,4632,539,3101,34,37,4645,8234, \\
1067,1579,566,4150,572,575,65,4161,6209,2116,3145,1610,1099,1100,2637,3658,3663,81,1617,602,4701,96, \\
1632,2144,99,2658,2659,1639,3178,6250,6766,3185,5234,2677,2678,3703,120,634,3706,640,129,642,2690,2691, \\
\text { 141, } 144,150,151,3736,3737,3742,1185,162,675,676,169,681,2733,177,180,694,184,697,2748,191,4799,198,199, \\
\text { 200, 201, 1222, 3784, 204, 3789, 206, 1742, 4304, 4817, 213, 2262, 5845, 217, 8409, 1243, 4317, 5341, 225, 3809, 227, 6370, } \\
742,5353,746,747,1261,241,3827,244,2292,1787,252,5883,772,3844,4357,6918,7428,6922,3851,1293,1298,275, \\
4370,2330,4380,1309,286,4384,4385,804,2344,4908,4397,2350,2352,5938,1332,1334,312,2362,5435,320,832, \\
\text { 2370, 323, 2888, 330, 843, 6474, 335, 1360, 1361, 3919, 2909, 350, 2910, 864, 4445, 3946, 1388, 7022, 1903, 1395, 7027, } \\
\text { 3957, 3958, 375, 4473, 379, 892, 382, 3972, 1928, 4490, 6028, 3986, 6035, 3995, 4510, 4511, 417, 1447, 1960, 4007, 4009,, } \\
1451,2479,1456,1459,3508,1975,440,953,2489,1980,446,448,1985,451,452,4552,1993,1994,460,1997,463,1496, \\
2016,4577,7651,2023,8681,499,4083,4596,3576,6141,510,1,2,3,5,7,9,10,11,13,14,15,16,17,19,20,21,25,26, \\
27,28,29,30,31,32,33,35,36,38,39,40,41,42,43,44,45,46,47,48,49,51,52,53,54,55,56,57,58,59,60,61,62,63, \\
64,66,67,68,70,71,72,73,74,75,76,77,78,79,80,82,84,85,86,87,88,89,91,92,93,94,95,97,98\end{array}$ \\
\hline & Test(14) & $385,579,4,388,583,142,334,144,145,432,19,462,22,23$ \\
\hline \multirow[t]{2}{*}{ NR-PPAR-gamma } & $\begin{array}{c}\text { Training } \\
\text { (293) }\end{array}$ & $\begin{array}{l}769,3,7,1801,12,3858,1812,24,536,3100,30,1827,3109,3111,3879,296,558,3892,823,1093,583,3143,329,1617, \\
82,84,596,342,2138,3935,96,97,1375,866,355,5474,5483,5484,3181,1904,2417,2166,886,3192,125,129,130, \\
391,1418,4490,4492,1165,658,4499,6293,7830,4247,2965,153,154,155,3226,159,1698,1187,164,418,2219,172, \\
173,3755,943,7351,4280,6330,6075,188,190,455,712,205,206,8154,4062,3807,2018,228,487,3048,3306,3307, \\
241,252,3839,0,1,2,5,6,8,9,10,11,14,15,18,19,20,21,22,23,26,28,29,31,32,33,34,35,36,37,39,40,41,46,47, \\
48,50,51,52,54,55,56,57,58,59,60,62,63,65,66,68,69,70,73,74,75,78,79,80,81,83,85,86,87,88,90,91,92, \\
95,98,100,101,102,103,104,105,107,108,109,111,113,114,116,117,118,119,120,121,122,124,126,127,128,131, \\
132,133,134,135,136,137,138,139,141,142,144,145,148,150,151,152,156,157,158,161,162,163,165,167,169, \\
170,171,174,175,176,177,179,180,181,182,183,184,185,186,187,189,191,192,194,195,196,197,198,199,200, \\
201,202,203,204,207,208,209,210,211,213,214,215,216,217,218,219,220,222,223,224,225,226,227,229,230, \\
231,232,233,234,235,236,237,238,239,240,242,244,245,246,247,248,249,250,251,254,255,256,257,259,260, \\
261,262,264,265,266,267,268,270\end{array}$ \\
\hline & Test(14) & $130,132,4,136,266,20,148,405,533,155,156,164,302,49$ \\
\hline \multirow[t]{2}{*}{ SR-ARE } & $\begin{array}{c}\text { Training } \\
(248)\end{array}$ & $\begin{array}{l}2049,2,3,8,2056,2057,4106,2061,16,2064,2065,4113,2071,2073,26,28,2079,2080,34,2084,2085,2086,39,40,41, \\
2091,2101,2046,63,2116,4165,2120,2123,2130,83,6228,90,91,96,98,99,2149,105,2156,2157,2158,4207,112, \\
2164,119,2172,2173,2174,126,2178,132,2181,139,141,2189,2192,4240,6289,148,149,2200,155,2207,4257,162, \\
163,2211,4263,2220,4272,177,6327,2233,189,2237,2240,193,2241,2242,2243,2246,202,203,205,2255,2262,2263, \\
2267,2269,2272,2274,2276,2277,2284,2285,2287,2289,243,4342,6391,4345,2298,2324,2327,2329,2330,293,2350, \\
2351,304,310,2359,318,320,332,2380,2382,2383,6488,2394,2397,4445,4449,2403,6501,360,2409,4456,363,4462, \\
4463,4467,373,2424,4474,2428,2429,2430,4479,2434,2437,2440,4488,2444,396,2447,2449,402,2451,2450,2457, \\
2459,412,2463,2465,2466,4516,4518,2471,2472,4519,2477,4525,2479,4526,4528,2485,438,2487,4534,4547,2502, \\
4550,457,4554,2508,461,4558,4559,2515,2524,4580,4581,4584,489,2537,2538,2539,2541,2545,2549,2550,4599, \\
4597,2553,6650,2555,4607,4608,2559,4610,2563,4612,4615,2568,2574,2579,2582,2583,6678,2586,539, \\
4587,\end{array}$ \\
\hline & Test(14) & $384,1,2,514,4,11,267,395,397,528,401,406,536,153$ \\
\hline \multirow[t]{2}{*}{ SR-ATAD5 } & $\begin{array}{c}\text { Training } \\
(310)\end{array}$ & $\begin{array}{l}514,5122,5,2566,12,1549,4622,2066,4628,1054,4639,4643,552,553,42,4651,4653,47,48,4656,2098,2105,1597, \\
6724,2118,584,4681,4171,2640,4689,4690,4691,4695,2137,93,94,1120,611,100,4707,4712,106,619,1134,113, \\
6771,122,4736,1156,1160,2195,664,1179,2204,2206,1185,675,2215,4775,2218,683,1710,2734,176,4790,183, \\
1209,1721,6329,191,192,4806,4807,200,718,2767,208,2256,2769,2259,219,735,737,6371,4836,230,742,233, \\
237,240,756,6390,6395,1276,253,1278,1279,257,770,262,777,1290,270,782,6415,6416,6419,3860,3861,6420, \\
3863,4887,6424,285,799,1313,807,3879,3883,3884,305,3889,2868,2872,2873,315,3899,317,318,2881,5954,835, \\
1347,4424,1354,3915,3916,333,341,1366,3934,3938,2403,6502,6503,876,2413,366,6511,3441,1401,1914,2426, \\
386,390,394,1931,3980,398,1422,3985,403,7060,3992,1433,922,3996,927,1441,4002,2467,938,2474,1455,1460, \\
4022,439,2491,452,1481,2507,5067,5068,462,4046,485,4596,4597,5109,4090,4092,0,1,2,3,4,6,7,8,11,13,15, \\
16,17,21,22,23,24,25,26,27,29,30,31,32,33,34,35,37,38,39,41,43,44,45,46,49,50,51,52,53,54,56,57,58,59, \\
60,61,62,63,64,65,66,67,68,69,70,71,75,76,78,79,80,81,83,84,85,86,87,89,90,91,92,96,97,98,99,101,102, \\
103,104,105,107,108,109,110,112,114,115,117,118,119,121,123,125,126,127,128,129,130,131,132,133,134,\end{array}$ \\
\hline & Test(15) & $2,5,8,10,140,13,525,271,529,18,19,282,157,542,175$ \\
\hline
\end{tabular}




\begin{tabular}{|c|c|c|}
\hline Dataset & & Omitted sample ID \\
\hline \multirow[t]{2}{*}{ SR-HSE } & $\begin{array}{c}\text { Training } \\
\text { (265) }\end{array}$ & $\begin{array}{l}3,4,4103,9,523,12,14,18,3092,23,2583,542,3102,7714,3619,3109,1574,551,43,3116,557,3118,5680,4657,50, \\
2616,5689,2618,2619,3132,575,65,66,2631,72,6216,2635,80,2640,2643,85,2134,2136,2137,2140,2141,2653, \\
2143,2144,2145,2655,2656,2660,2149,2150,3166,2152,2153,2154,2156,109,2158,2161,2162,2675,2164,2678, \\
2679,2168,2169,2170,5753,2686,5246,3206,2184,2187,2191,1681,3732,2198,3735,3739,6301,3231,2720,2209, \\
1186,4255,2216,2218,3756,5806,2735,3764,2229,2230,3765,3766,193,2241,2242,2243,2753,3784,2250,2251, \\
2765,2260,2262,6873,3805,4322,2279,3816,3818,2284,2286,2289,2290,6386,3316,3830,2295,2301,2813,2303, \\
3839,2307,2309,2311,2314,2320,2321,3858,5905,797,2847,6439,2344,2350,5427,2361,5438,2367,3392,2369, \\
3906,5441,5444,2377,5458,2388,2391,2392,5464,2395,2396,3931,3932,2400,3937,2404,357,2405,2412,3949, \\
2414,2417,5491,4980,5494,5496,2425,2433,5508,2441,5514,5516,7564,2446,7565,2451,404,5524,406,407,6085, \\
2974,6087,2980,421,423,2985,2474,5545,2989,2990,2991,5550,2995,437,439,440,441,3001,2492,3004,453,454, \\
966,2501,4040,5573,3531,460,973,3020,3022,5575,3025,6103,2525,481,3043,2535,488,4073,3054,5616,499, \\
2558,511,0,1,2,7,8,11,15,16,17,21,22,24,25,26,27,29,32,33,35,36,37,38,39,40,41,42,44,45,46,48,49,51\end{array}$ \\
\hline & Test(15) & $131,4,581,357,139,559,400,305,464,595,20,602,407,314,348$ \\
\hline \multirow[t]{2}{*}{ SR-MMP } & $\begin{array}{c}\text { Training } \\
\text { (243) }\end{array}$ & $\begin{array}{l}4099,516,4100,4614,520,3085,6670,529,3089,1555,3604,4628,3095,1048,1050,3616,3622,3623,40,41,7215,5168, \\
4657,3634,563,2619,5179,7228,7233,1092,3140,3655,3656,592,593,3666,595,3667,7253,3670,1624,3672,4696, \\
1116,4708,3173,1638,2664,3179,5739,621,3693,5740,2160,5744,630,4731,637,127,3723,3724,3727,2196,3734, \\
4759,5272,669,4767,675,4778,3756,4781,1711,6832,1201,192,193,3264,3776,708,1734,3271,3277,719,4815, \\
1752,223,735,1252,1766,1261,753,4366,1295,785,280,282,797,5920,801,3363,5412,3366,296,301,4397,4914, \\
820,1333,1846,5429,312,315,2883,3397,4934,3400,329,1869,335,848,2897,3408,339,3412,4948,5974,3415,345, \\
3421,1374,2910,1376,3426,1380,4966,4977,4978,884,1398,1399,2940,895,1921,1922,6018,1415,2440,2951,3465, \\
2958,2959,3473,402,2968,409,3480,2971,3484,5018,415,417,929,2977,2984,426,3498,6058,2989,1454,945,1969, \\
947,3507,4529,4024,3515,4027,447,7105,450,3522,1476,4046,5582,5587,4564,469,4572,3549,479,481,3045, \\
489,5609,5611,1516,3059,6643,503,14,17,25,65,67,68,95,105,109,121,138,139,153,173,187,188,191,196,197, \\
201,204,205,206,219,221,225,227,229,234,237,240,253,254,256,257,258,261,262,274,276,283,286,290\end{array}$ \\
\hline & Test(12) & $256,1,3,4,387,8,16,528,535,536,153,411$ \\
\hline \multirow[t]{2}{*}{ SR-p53 } & $\begin{array}{c}\text { Training } \\
\text { (277) }\end{array}$ & $\begin{array}{l}0,4103,10,12,14,16,17,18,2064,20,21,2067,23,26,29,30,31,2079,33,2077,35,36,2090,43,45,2093,47,48,49, \\
4147,4148,57,4154,2107,4156,61,64,6208,66,67,68,2117,70,71,73,2122,75,79,80,4176,4178,2132,87,91,94,97, \\
2153,106,109,2158,110,111,4205,115,2165,118,121,123,124,125,129,130,2180,4228,137,2188,141,2191,145, \\
146,147,2195,2196,150,154,157,158,159,162,164,167,170,172,173,174,175,177,180,184,2238,197,202,205, \\
2253,207,211,214,4313,219,2268,226,230,6375,233,237,241,242,250,4346,253,4349,4354,262,268,271,273, \\
2326,279,280,2327,282,287,291,309,313,315,316,318,2368,324,327,2377,330,335,339,341,346,349,351,360, \\
2411,371,372,375,382,384,386,389,390,393,394,2441,400,414,2465,418,2468,422,2470,430,2478,8625,434,438, \\
445,447,459,462,478,480,492,499,500,501,508,513,514,515,516,518,519,522,534,547,549,550,553,555,559, \\
560,4655,4667,2620,573,575,2625,581,583,594,595,599,602,604,607,612,620,624,630,642,647,663,4775,694, \\
695,726,769,772,776,799,808,826,831,842,847,849,2897,861,865,868,870,871,873,886,890,909,5022,932,951, \\
955,961,3012,972,974,994,1010,1028,1031,1035,1054,1055,1065,1068,1072,1077,1085,1107,1124,3174,1129, \\
1134,1137,1138,1159,1160,1184,1192,1215\end{array}$ \\
\hline & Test(14) & $3,4,5,134,6,8,265,521,16,17,274,19,20,21$ \\
\hline
\end{tabular}

Table S14. Comparison of prediction results of GBDT with five different molecular fingerprints in toxicity prediction before data preprocessing.

\begin{tabular}{|l|c|c|c|c|c|c|c|c|c|c|}
\hline \multirow{3}{*}{ Data set } & \multicolumn{10}{|c|}{ Molecular fingerprints } \\
\cline { 2 - 13 } & \multicolumn{2}{|c|}{ Estate 2 } & \multicolumn{2}{c|}{ Estate 1 } & \multicolumn{2}{c|}{ Maccs } & \multicolumn{2}{c|}{ MWCG } & \multicolumn{2}{c|}{ hybrid } \\
\cline { 2 - 12 } & AUC & ACC & AUC & ACC & AUC & ACC & AUC & ACC & AUC & ACC \\
\hline NR-AhR & 0.829 & 0.877 & 0.870 & 0.892 & 0.861 & 0.892 & $\mathbf{0 . 8 9 3}$ & 0.890 & 0.886 & 0.890 \\
\hline NR-AR & 0.782 & 0.978 & $\mathbf{0 . 7 8 6}$ & 0.979 & 0.756 & 0.976 & 0.719 & 0.983 & 0.776 & 0.979 \\
\hline NR-AR-LBD & 0.619 & 0.983 & 0.666 & 0.981 & $\mathbf{0 . 7 7 4}$ & 0.988 & 0.693 & 0.981 & 0.709 & 0.981 \\
\hline NR-Aromatase & 0.780 & 0.922 & 0.776 & 0.926 & 0.779 & 0.928 & 0.753 & 0.928 & $\mathbf{0 . 7 9 8}$ & 0.928 \\
\hline NR-ER & 0.782 & 0.911 & 0.752 & 0.895 & 0.761 & 0.913 & 0.800 & 0.915 & $\mathbf{0 . 8 0 1}$ & 0.905 \\
\hline NR-ER-LBD & $\mathbf{0 . 8 3 5}$ & 0.957 & 0.764 & 0.963 & 0.788 & 0.958 & 0.749 & 0.963 & 0.762 & 0.967 \\
\hline NR-PPAR-gamma & 0.739 & 0.947 & 0.805 & 0.949 & $\mathbf{0 . 8 1 3}$ & 0.950 & 0.695 & 0.949 & 0.741 & 0.952 \\
\hline SR-ARE & 0.753 & 0.821 & 0.746 & 0.814 & 0.757 & 0.818 & 0.747 & 0.814 & $\mathbf{0 . 7 8 7}$ & 0.832 \\
\hline SR-ATAD5 & 0.743 & 0.937 & 0.770 & 0.939 & 0.734 & 0.940 & 0.715 & 0.936 & $\mathbf{0 . 7 8 6}$ & 0.931 \\
\hline SR-HSE & 0.744 & 0.952 & 0.819 & 0.954 & $\mathbf{0 . 8 7 3}$ & 0.962 & 0.789 & 0.962 & 0.846 & 0.962 \\
\hline SR-MMP & 0.905 & 0.908 & 0.922 & 0.893 & 0.907 & 0.894 & $\mathbf{0 . 9 3 6}$ & 0.920 & 0.930 & 0.904 \\
\hline SR-p53 & 0.745 & 0.919 & 0.767 & 0.924 & 0.808 & 0.932 & 0.816 & 0.935 & $\mathbf{0 . 8 2 9}$ & 0.924 \\
\hline
\end{tabular}


Table S15. The hyper-parameters in GBDT algorithm with five different fingerprints for 12 datasets in toxicity prediction before data preprocessing.

\begin{tabular}{|c|c|c|c|c|c|c|c|c|c|c|c|}
\hline Dataset & $\begin{array}{l}\text { finger } \\
\text { print }\end{array}$ & $\begin{array}{l}n \text { es- } \\
\text { tima- } \\
\text { tors }\end{array}$ & $\begin{array}{l}\max \\
\text { depth }\end{array}$ & $\begin{array}{l}\text { min } \\
\text { sam- } \\
\text { ple } \\
\text { split }\end{array}$ & $\begin{array}{l}\text { learning } \\
\text { rate }\end{array}$ & Dataset & $\begin{array}{l}\text { finger } \\
\text { print }\end{array}$ & $\begin{array}{l}n \text { es- } \\
\text { tima- } \\
\text { tors }\end{array}$ & $\begin{array}{l}\max \\
\text { depth }\end{array}$ & $\begin{array}{l}\text { min } \\
\text { sam- } \\
\text { ple } \\
\text { split }\end{array}$ & $\begin{array}{l}\text { learning } \\
\text { rate }\end{array}$ \\
\hline \multirow{5}{*}{$\begin{array}{l}\text { NR- } \\
\text { AhR }\end{array}$} & Estate 2 & 8000 & 5 & 2 & 0.009 & \multirow{5}{*}{$\begin{array}{l}\text { NR- } \\
\text { ppar- } \\
\text { gamma }\end{array}$} & Estate 2 & 10100 & 3 & 2 & 0.001 \\
\hline & Estate 1 & 8000 & 3 & 2 & 0.01 & & Estate 1 & 10000 & 3 & 2 & 0.0004 \\
\hline & MACCS & 8000 & 3 & 2 & 0.003 & & MACCS & 10000 & 3 & 2 & 0.006 \\
\hline & MWCG & 10000 & 3 & 3 & 0.005 & & MWCG & 10000 & 3 & 3 & 0.005 \\
\hline & Hybrid & 8000 & 9 & 8 & 0.02 & & Hybrid & 15000 & 9 & 4 & 0.015 \\
\hline \multirow{5}{*}{ NR-AR } & Estate 2 & 8000 & 3 & 2 & 0.002 & \multirow{5}{*}{$\begin{array}{l}\text { SR- } \\
\text { ARE }\end{array}$} & Estate 2 & 10000 & 11 & 2 & 0.006 \\
\hline & Estate 1 & 8000 & 3 & 2 & 0.004 & & Estate 1 & 10100 & 7 & 2 & 0.001 \\
\hline & MACCS & 8000 & 3 & 2 & 0.0004 & & MACCS & 10100 & 3 & 2 & 0.007 \\
\hline & MWCG & 15000 & 7 & 6 & 0.005 & & MWCG & 8000 & 3 & 4 & 0.02 \\
\hline & Hybrid & 15000 & 9 & 6 & 0.015 & & Hybrid & 15000 & 9 & 8 & 0.02 \\
\hline \multirow{5}{*}{$\begin{array}{l}\text { NR- } \\
\text { AR- } \\
\text { LBD }\end{array}$} & Estate 2 & 10000 & 3 & 2 & 0.03 & \multirow{5}{*}{$\begin{array}{l}\text { SR- } \\
\text { ATAD5 }\end{array}$} & Estate 2 & 10000 & 3 & 2 & 0.002 \\
\hline & Estate 1 & 10100 & 3 & 2 & 0.005 & & Estate 1 & 10100 & 3 & 4 & 0.001 \\
\hline & MACCS & 10100 & 3 & 2 & 0.03 & & MACCS & 10100 & 3 & 2 & 0.0005 \\
\hline & MWCG & 8000 & 5 & 3 & 0.005 & & MWCG & 12000 & 7 & 6 & 0.008 \\
\hline & Hybrid & 15000 & 5 & 4 & 0.015 & & Hybrid & 15000 & 7 & 2 & 0.008 \\
\hline \multirow{5}{*}{$\begin{array}{l}\text { NR- } \\
\text { Arom } \\
\text { atase }\end{array}$} & Estate 2 & 10100 & 3 & 2 & 0.0008 & \multirow{5}{*}{$\begin{array}{l}\text { SR- } \\
\text { HSE }\end{array}$} & Estate 2 & 10000 & 3 & 2 & 0.003 \\
\hline & Estate 1 & 15000 & 3 & 2 & 0.001 & & Estate 1 & 10100 & 6 & 2 & 0.0007 \\
\hline & MACCS & 10100 & 5 & 2 & 0.0006 & & MACCS & 10100 & 3 & 2 & 0.006 \\
\hline & MWCG & 15000 & 7 & 2 & 0.008 & & MWCG & 12000 & 7 & 9 & 0.005 \\
\hline & Hybrid & 8000 & 9 & 2 & 0.015 & & Hybrid & 8000 & 3 & 8 & 0.008 \\
\hline \multirow{5}{*}{ NR-ER } & Estate 2 & 10100 & 3 & 2 & 0.0008 & \multirow{5}{*}{$\begin{array}{l}\text { SR- } \\
\text { MMP }\end{array}$} & Estate 2 & 10100 & 3 & 2 & 0.008 \\
\hline & Estate 1 & 10100 & 3 & 2 & 0.003 & & Estate 1 & 10100 & 5 & 4 & 0.006 \\
\hline & MACCS & 10100 & 3 & 2 & 0.003 & & MACCS & 10100 & 3 & 2 & 0.006 \\
\hline & MWCG & 10000 & 3 & 3 & 0.005 & & MWCG & 10000 & 7 & 3 & 0.01 \\
\hline & Hybrid & 8000 & 7 & 4 & 0.02 & & Hybrid & 8000 & 9 & 6 & 0.008 \\
\hline \multirow{5}{*}{$\begin{array}{l}\text { NR- } \\
\text { ER- } \\
\text { LBD }\end{array}$} & Estate 2 & 10100 & 5 & 2 & 0.0008 & \multirow{5}{*}{ SR-p53 } & Estate 2 & 10100 & 10 & 6 & 0.008 \\
\hline & Estate 1 & 10100 & 3 & 2 & 0.001 & & Estate 1 & 10100 & 5 & 2 & 0.001 \\
\hline & MACCS & 10100 & 3 & 2 & 0.006 & & MACCS & 10100 & 5 & 2 & 0.003 \\
\hline & MWCG & 10000 & 7 & 6 & 0.005 & & MWCG & 8000 & 5 & 3 & 0.005 \\
\hline & Hybrid & 12000 & 9 & 4 & 0.008 & & Hybrid & 12000 & 3 & 6 & 0.008 \\
\hline
\end{tabular}

\section{References}

[1] Alexios Koutsoukas, Joseph St Amand, Meenakshi Mishra, and Jun Huan. Predictive toxicology: modeling chemical induced toxicological response combining circular fingerprints with random forest and support vector machine. Frontiers in Environmental Science, 4:11, 2016.

[2] Jin Zhang, Daniel Mucs, Ulf Norinder, and Fredrik Svensson. Lightgbm: An effective and scalable algorithm for prediction of chemical toxicity-application to the tox 21 and mutagenicity data sets. Journal of Chemical Information and Modeling, 59(10):4150-4158, 2019.

[3] Abdul Karim, Avinash Mishra, MA Hakim Newton, and Abdul Sattar. Efficient toxicity prediction via simple features using shallow neural networks and decision trees. ACS Omega, 4(1):1874-1888, 2019.

[4] Andreas Mayr, Günter Klambauer, Thomas Unterthiner, and Sepp Hochreiter. Deeptox: toxicity prediction using deep learning. Frontiers in Environmental Science, 3:80, 2016.

[5] Priyanka Banerjee, Vishal B Siramshetty, Malgorzata N Drwal, and Robert Preissner. Computational methods for prediction of in vitro effects of new chemical structures. Journal of cheminformatics, 8(1):111, 2016. 
[6] Stephen J Capuzzi, Regina Politi, Olexandr Isayev, Sherif Farag, and Alexander Tropsha. Qsar modeling of tox 21 challenge stress response and nuclear receptor signaling toxicity assays. Frontiers in Environmental Science, 4:3, 2016. 